\title{
$\rho \rho$ scattering revisited with coupled channels of pseudoscalar mesons
}

\author{
Zheng-Li Wang ${ }^{1,2, *}$ and Bing-Song Zou ${ }^{1,2,3, \dagger}$ \\ ${ }^{1}$ CAS Key Laboratory of Theoretical Physics, Institute of Theoretical Physics, \\ Chinese Academy of Sciences, Beijing 100190,China \\ ${ }^{2}$ University of Chinese Academy of Sciences (UCAS), Beijing 100049, China \\ ${ }^{3}$ Central South University, Hunan 410083, China
}

(Received 30 January 2019; published 15 May 2019)

\begin{abstract}
The $\rho \rho$ scattering has been studied by two groups which both claimed a dynamical generated scalar meson, most likely to be $f_{0}(1370)$. Here we investigate the influence of coupled channels of pseudoscalar mesons, i.e., $\pi \pi$ and $\bar{K} K$, on this dynamical generated scalar state. With the coupled-channel effect included, the pole and partial decay widths are found to be more close to PDG values for $f_{0}(1500)$.
\end{abstract}

DOI: $10.1103 /$ PhysRevD.99.096014

\section{INTRODUCTION}

The chiral unitary approach, which has made much progress in the study of pseudoscalar meson-meson [1] and meson-baryon [2,3] interactions, has been used to study the interaction of vector mesons among themselves. The first such study of the $S$-wave $\rho \rho$ interactions found that the $f_{0}(1370)$ and the $f_{2}(1270)$ could be dynamically generated [4]. The work found that the strength of the attractive interaction in the tensor channel is much stronger than that in the scalar channel, hence leads to a tighter bound tensor state than the corresponding scalar one.

The work [4] is based on the assumption that the three momenta of the $\rho$ is negligibly small compared to its large mass. This assumption was questioned by a recent work [5], which pointed out the importance of relativistic effect for energies around $f_{2}(1270)$ well below the $\rho \rho$ threshold. The $N / D$ method [6-10] was used to get the partial-wave amplitudes, which results in a pole for the scalar state similar to Ref. [4] but no pole for any tensor state in contradiction with Ref. [4]. However, this conclusion was not agreed upon by Ref. [11] in which the nonrelativistic assumption was dropped by evaluating exactly the loops with full relativistic $\rho$ propagators in solving the B-S equation for $\rho \rho$ scattering. Both the scalar state and tensor state associated with $f_{0}(1370)$ and $f_{2}(1270)$, respectively, were found in consistence with the conclusion of Ref. [4].

From the studies of the above two groups, obviously, for the energies around $f_{2}(1270)$ well below $\rho \rho$ threshold,

\footnotetext{
wangzhengli@itp.ac.cn

†oubs@itp.ac.cn
}

Published by the American Physical Society under the terms of the Creative Commons Attribution 4.0 International license. Further distribution of this work must maintain attribution to the author(s) and the published article's title, journal citation, and DOI. Funded by SCOAP ${ }^{3}$. there is strong model dependence for the interaction of two far off-mass-shell $\rho$ mesons. For the scalar state closer to the $\rho \rho$ threshold, the two groups got similar results rather model independently. In this paper, we shall study the influence of coupled channels of pseudoscalar mesons, i.e., $\pi \pi$ and $\bar{K} K$, on this dynamical generated scalar state. In the $\rho \rho-K \bar{K}$ coupling we consider the case of $K$ and $K^{*}$ exchange, while in the $\rho \rho-\pi \pi$ coupling we consider the case of $\pi$ and $\omega$ exchange.

\section{FORMALISM}

\section{A. $\rho \rho \rightarrow \pi \pi$ with $\pi$ exchange}

We investigate the coupled-channel effect based on a chiral covariant framework [5]. We follow the formalism of the hidden gauge interaction which provides the $\rho \pi \pi$ coupling by means of the Lagrangian $[12,13]$

$$
\mathcal{L}_{V P P}=-i g\left\langle V^{\mu}\left[P, \partial_{\mu} P\right]\right\rangle,
$$

where the symbol $\langle\ldots\rangle$ stands for the trace in the $S U(3)$ space with the coupling constant $g=M_{V} / 2 f_{\pi}$ with $M_{V}$ the mass of the vector meson and $f_{\pi}=93 \mathrm{MeV}$ the pion decay constant. The matrices $V_{\mu}$ and $P$ are given by

$$
\begin{aligned}
V_{\mu} & =\left(\begin{array}{ccc}
\frac{\rho_{0}}{\sqrt{2}}+\frac{\omega}{\sqrt{2}} & \rho^{+} & K^{*+} \\
\rho^{-} & -\frac{\rho_{0}}{\sqrt{2}}+\frac{\omega}{\sqrt{2}} & K^{* 0} \\
K^{*-} & \bar{K}^{* 0} & \phi
\end{array}\right)_{\mu} \\
P & =\left(\begin{array}{ccc}
\frac{\pi^{0}}{\sqrt{2}}+\frac{\eta_{8}}{\sqrt{6}} & \pi^{+} & K^{+} \\
\pi^{-} & -\frac{\pi^{0}}{\sqrt{2}}+\frac{\eta_{8}}{\sqrt{6}} & K^{0} \\
K^{-} & \bar{K}^{0} & -\frac{2 \eta_{8}}{\sqrt{6}}
\end{array}\right) .
\end{aligned}
$$

To get the three different isospin amplitudes for $\rho \rho \rightarrow \pi \pi$ we need the knowledge of the transitions $\rho^{+}\left(p_{1}\right) \rho^{-}\left(p_{2}\right) \rightarrow$ $\pi^{+}\left(p_{3}\right) \pi^{-}\left(p_{4}\right), \rho^{+}\left(p_{1}\right) \rho^{-}\left(p_{2}\right) \rightarrow \pi^{0}\left(p_{3}\right) \pi^{0}\left(p_{4}\right)$, etc. 


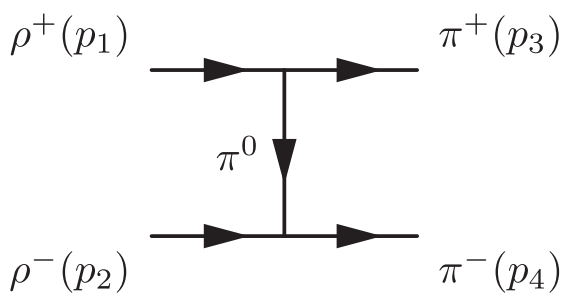

FIG. 1. $\pi$-exchange diagram for $\rho^{+} \rho^{-} \rightarrow \pi^{+} \pi^{-}$.

Starting with the Lagrangian in Eq. (1) we can immediately obtain the amplitude $A_{t}\left(p_{1}, p_{2}, p_{3}, p_{4}\right)$ of $\rho^{+}\left(p_{1}\right) \rho^{-}\left(p_{2}\right) \rightarrow \pi^{+}\left(p_{3}\right) \pi^{-}\left(p_{4}\right)$ corresponding to Fig. 1 as

$A_{t}\left(p_{1}, p_{2}, p_{3}, p_{4}\right)=\frac{-8 g^{2}}{\left(p_{1}-p_{3}\right)^{2}-m_{\pi}^{2}} \epsilon_{1} \cdot p_{3} \epsilon_{2} \cdot p_{4}$.

In this equation, the $\epsilon_{i}$ corresponds to the polarization vector of the $i$ th $\rho$. Each polarization vector is characterized by its three-momentum $\mathbf{p}_{i}$ and third component of the spin $\sigma_{i}$. Explicit expressions of these polarization vectors are given by [5]

$$
\begin{gathered}
\epsilon(\mathbf{p}, 0)=\left(\begin{array}{c}
\gamma \beta \cos \theta \\
\frac{1}{2}(\gamma-1) \sin 2 \theta \cos \phi \\
\frac{1}{2}(\gamma-1) \sin 2 \theta \sin \phi \\
\frac{1}{2}(1+\gamma+(\gamma-1) \cos 2 \theta)
\end{array}\right), \\
\epsilon(\mathbf{p}, \pm 1)=\mp \frac{1}{\sqrt{2}}\left(\begin{array}{c}
\gamma \beta e^{ \pm i \phi} \sin \theta \\
1+(\gamma-1) e^{ \pm i \phi} \sin ^{2} \theta \cos \phi \\
\pm i+(\gamma-1) e^{ \pm i \phi} \sin ^{2} \theta \sin \phi \\
\frac{1}{2}(\gamma-1) e^{ \pm i \phi} \sin 2 \theta
\end{array}\right),
\end{gathered}
$$

where $\beta=|\mathbf{p}| / E_{p}, \gamma=1 / \sqrt{1-\beta^{2}}$, and $\theta$ and $\phi$ are the polar angle and azimuthal angle of $\mathbf{p}$, respectively. The $u$ channel $\pi$-exchange amplitude $A_{u}$ can be obtained from the expression of $A_{t}$ by exchanging $p_{3} \leftrightarrow p_{4}$. In this way,

$$
A_{u}\left(p_{1}, p_{2}, p_{3}, p_{4}\right)=A_{t}\left(p_{1}, p_{2}, p_{4}, p_{3}\right) .
$$

And now we write the tree-level amplitude for $\rho \rho \rightarrow \pi \pi$ with the $\pi$ exchange

$$
\begin{aligned}
\rho^{+}\left(p_{1}\right) \rho^{-}\left(p_{2}\right) & \rightarrow \pi^{+}\left(p_{3}\right) \pi^{-}\left(p_{4}\right): A_{t}, \\
\rho^{+}\left(p_{1}\right) \rho^{-}\left(p_{2}\right) & \rightarrow \pi^{0}\left(p_{3}\right) \pi^{0}\left(p_{4}\right): A_{t}+A_{u}, \\
\rho^{0}\left(p_{1}\right) \rho^{0}\left(p_{2}\right) & \rightarrow \pi^{+}\left(p_{3}\right) \pi^{-}\left(p_{4}\right): A_{t}+A_{u}, \\
\rho^{0}\left(p_{1}\right) \rho^{0}\left(p_{2}\right) & \rightarrow \pi^{0}\left(p_{3}\right) \pi^{0}\left(p_{4}\right): 0 .
\end{aligned}
$$

In order to obtain the $S$-wave amplitude in the isospin $I=0$ channel we need the isospin eigenstates. We have

$$
\begin{aligned}
|\rho \rho, I=0\rangle= & -\frac{1}{\sqrt{3}} \mid \rho^{+}\left(p_{1}\right) \rho^{-}\left(p_{2}\right)+\rho^{-}\left(p_{1}\right) \rho^{+}\left(p_{2}\right) \\
& \left.+\rho^{0}\left(p_{1}\right) \rho^{0}\left(p_{2}\right)\right\rangle, \\
|\pi \pi, I=0\rangle= & -\frac{1}{\sqrt{3}} \mid \pi^{+}\left(p_{1}\right) \pi^{-}\left(p_{2}\right)+\pi^{-}\left(p_{1}\right) \pi^{+}\left(p_{2}\right) \\
& \left.+\pi^{0}\left(p_{1}\right) \pi^{0}\left(p_{2}\right)\right\rangle,
\end{aligned}
$$

where we have used the convention $\left|\rho^{+}\right\rangle=-|1,1\rangle$ and $\left|\pi^{+}\right\rangle=-|1,1\rangle$ of the isospin. By taking into account Eq. (7) and the amplitudes in Eq. (6) we can now write the isospin $I=0$ amplitude for $\rho \rho \rightarrow \pi \pi$

$$
T_{\pi}^{(0)}=16 g^{2}\left(\frac{\epsilon_{1} \cdot p_{3} \epsilon_{2} \cdot p_{4}}{m_{\pi}^{2}-t}+\frac{\epsilon_{1} \cdot p_{4} \epsilon_{2} \cdot p_{3}}{m_{\pi}^{2}-u}\right),
$$

where $t=\left(p_{1}-p_{3}\right)^{2}$ and $u=\left(p_{1}-p_{4}\right)^{2}$.

\section{B. $\rho \rho \rightarrow \pi \pi$ with $\omega$ exchange}

One needs the $\rho \omega \pi$ coupling which is provided within the framework [14] of the hidden gauge formalism by means of the Lagrangian

$$
\mathcal{L}_{V V P}=\frac{G^{\prime}}{\sqrt{2}} \epsilon^{\mu \nu \alpha \beta}\left\langle\partial_{\mu} V_{\nu} \partial_{\alpha} V_{\beta} P\right\rangle
$$

with

$$
G^{\prime}=\frac{3 g^{\prime 2}}{4 \pi^{2} f_{\pi}} \quad g^{\prime}=-\frac{G_{V} m_{\rho}}{\sqrt{2} f_{\pi}^{2}},
$$

where $G_{V}=55 \mathrm{MeV}$ and $f_{\pi}=93 \mathrm{MeV}$. At this point we can write down the amplitude of $\rho^{+}\left(p_{1}\right) \rho^{-}\left(p_{2}\right) \rightarrow$ $\pi^{+}\left(p_{3}\right) \pi^{-}\left(p_{4}\right)$ with the $\omega$ exchange corresponding to Fig. 2 as in the $\pi$-exchange case

$$
\begin{aligned}
B_{t}= & \frac{-G^{2}}{\left(p_{1}-p_{3}\right)^{2}-m_{\omega}^{2}}\left(p_{3} \cdot p_{4} p_{1} \cdot \epsilon_{2} p_{2} \cdot \epsilon_{1}\right. \\
& +p_{1} \cdot p_{2} p_{4} \cdot \epsilon_{1} p_{3} \cdot \epsilon_{2}+p_{1} \cdot p_{4} p_{2} \cdot p_{3} \epsilon_{1} \cdot \epsilon_{2} \\
& -p_{2} \cdot p_{3} p_{1} \cdot \epsilon_{2} p_{4} \cdot \epsilon_{1}-p_{1} \cdot p_{4} p_{2} \cdot \epsilon_{1} p_{3} \cdot \epsilon_{2} \\
& \left.-p_{1} \cdot p_{2} p_{3} \cdot p_{4} \epsilon_{1} \cdot \epsilon_{2}\right) .
\end{aligned}
$$

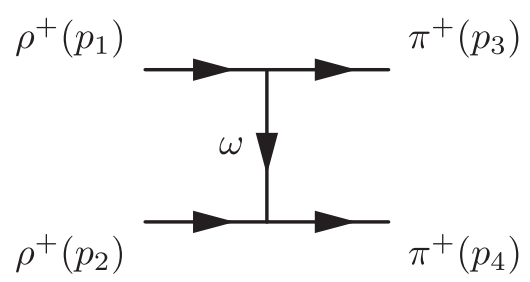

FIG. 2. $\omega$-exchange diagram for $\rho^{+} \rho^{-} \rightarrow \pi^{+} \pi^{-}$. 
And the $u$-channel $\omega$-exchange amplitude $B_{u}\left(p_{1}, p_{2}\right.$, $\left.p_{3}, p_{4}\right)$ can be obtained from the expression of $B_{t}$ as the case in the $\pi$ exchange by exchanging $p_{3} \leftrightarrow p_{4}$, thus

$$
B_{u}\left(p_{1}, p_{2}, p_{3}, p_{4}\right)=B_{t}\left(p_{1}, p_{2}, p_{4}, p_{3}\right) .
$$

Next we write the tree-level amplitude for $\rho \rho \rightarrow \pi \pi$ with the $\omega$ exchange

$$
\begin{aligned}
\rho^{+}\left(p_{1}\right) \rho^{-}\left(p_{2}\right) & \rightarrow \pi^{+}\left(p_{3}\right) \pi^{-}\left(p_{4}\right): B_{t}, \\
\rho^{+}\left(p_{1}\right) \rho^{-}\left(p_{2}\right) & \rightarrow \pi^{0}\left(p_{3}\right) \pi^{0}\left(p_{4}\right): 0, \\
\rho^{0}\left(p_{1}\right) \rho^{0}\left(p_{2}\right) & \rightarrow \pi^{+}\left(p_{3}\right) \pi^{-}\left(p_{4}\right): 0, \\
\rho^{0}\left(p_{1}\right) \rho^{0}\left(p_{2}\right) & \rightarrow \pi^{0}\left(p_{3}\right) \pi^{0}\left(p_{4}\right): B_{t}+B_{u} .
\end{aligned}
$$

Then, using Eq. (7) we can get the $I=0$ amplitude

$$
\begin{aligned}
T_{\omega}^{(0)}= & \frac{-G^{\prime 2}}{\left(p_{1}-p_{3}\right)^{2}-m_{\omega}^{2}}\left(p_{3} \cdot p_{4} p_{1} \cdot \epsilon_{2} p_{2} \cdot \epsilon_{1}\right. \\
& +p_{1} \cdot p_{2} p_{4} \cdot \epsilon_{1} p_{3} \cdot \epsilon_{2}+p_{1} \cdot p_{4} p_{2} \cdot p_{3} \epsilon_{1} \cdot \epsilon_{2} \\
& -p_{2} \cdot p_{3} p_{1} \cdot \epsilon_{2} p_{4} \cdot \epsilon_{1}-p_{1} \cdot p_{4} p_{2} \cdot \epsilon_{1} p_{3} \cdot \epsilon_{2} \\
& \left.-p_{1} \cdot p_{2} p_{3} \cdot p_{4} \epsilon_{1} \cdot \epsilon_{2}\right)+\left(p_{3} \leftrightarrow p_{4}\right) .
\end{aligned}
$$

\section{C. $\rho \rho \rightarrow K \bar{K}$ with $K$ exchange}

The $\rho K K$ coupling is provided in the same Lagrangian in Eq. (1), so we can immediately write down the amplitude of $\rho^{+}\left(p_{1}\right) \rho^{-}\left(p_{2}\right) \rightarrow K^{+}\left(p_{3}\right) K^{-}\left(p_{4}\right)$ with the $K$ exchange corresponding to Fig. 3

$$
C_{t}\left(p_{1}, p_{2}, p_{3}, p_{4}\right)=-4 g^{2} \frac{\epsilon_{1} \cdot p_{3} \epsilon_{2} \cdot p_{4}}{\left(p_{1}-p_{3}\right)^{2}-m_{K}^{2}},
$$

and the $u$ channel

$$
C_{u}\left(p_{1}, p_{2}, p_{3}, p_{4}\right)=C_{t}\left(p_{1}, p_{2}, p_{4}, p_{3}\right)
$$

Then we can obtain the tree-level amplitudes for $\rho \rho \rightarrow K \bar{K}$ with the $K$ exchange as the following:

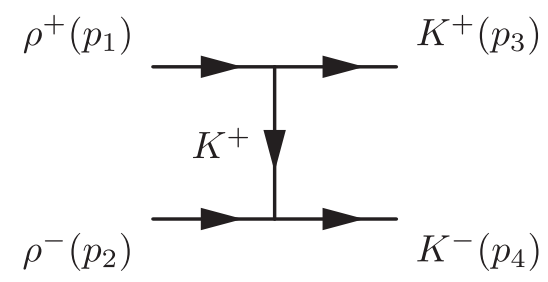

FIG. 3. $K$-exchange amplitude for $\rho^{+}\left(p_{1}\right) \rho^{-}\left(p_{2}\right) \rightarrow$ $K^{+}\left(p_{3}\right) K^{-}\left(p_{4}\right)$.

$$
\begin{aligned}
\rho^{+}\left(p_{1}\right) \rho^{-}\left(p_{2}\right) & \rightarrow K^{+}\left(p_{3}\right) K^{-}\left(p_{4}\right): C_{t}, \\
\rho^{+}\left(p_{1}\right) \rho^{-}\left(p_{2}\right) & \rightarrow K^{0}\left(p_{3}\right) \bar{K}^{0}\left(p_{4}\right): C_{u}, \\
\rho^{0}\left(p_{1}\right) \rho^{0}\left(p_{2}\right) & \rightarrow K^{+}\left(p_{3}\right) K^{-}\left(p_{4}\right): \frac{C_{t}+C_{u}}{2}, \\
\rho^{0}\left(p_{1}\right) \rho^{0}\left(p_{2}\right) & \rightarrow K^{0}\left(p_{3}\right) \bar{K}^{0}\left(p_{4}\right): \frac{C_{t}+C_{u}}{2} .
\end{aligned}
$$

Similar to Eq. (7) we need the isospin $I=0$ eigenstate for $|K \bar{K}\rangle$. We have

$$
|K \bar{K}\rangle=-\frac{1}{\sqrt{2}}\left|K^{+}\left(p_{1}\right) K^{-}\left(p_{2}\right)+K^{0}\left(p_{1}\right) \bar{K}^{0}\left(p_{2}\right)\right\rangle,
$$

where we use the convention $\left|K^{+}\right\rangle=-\left|\frac{1}{2}, \frac{1}{2}\right\rangle$ of the isospin. By using the isospin wave functions we can obtain for $I=0$

$$
T_{K}^{(0)}=2 \sqrt{6} g^{2}\left(\frac{\epsilon_{1} \cdot p_{3} \epsilon_{2} \cdot p_{4}}{m_{K}^{2}-t}+\frac{\epsilon_{1} \cdot p_{4} \epsilon_{2} \cdot p_{3}}{m_{K}^{2}-u}\right)
$$

with $t$ and $u$, the usual Mandelstam variable. We can see that Eq. (19) is similar to Eq. (8). The former can be obtained from the latter just by substituting $16 \rightarrow 2 \sqrt{6}$ and $m_{\pi} \rightarrow m_{K}$.

\section{D. $\rho \rho \rightarrow K \bar{K}$ with $K^{*}$ exchange}

As for the $\rho K K^{*}$ coupling, we use the Lagrangian in Eq. (9). Then we get the amplitude for $\rho^{+}\left(p_{1}\right) \rho^{-}\left(p_{2}\right) \rightarrow$ $K^{+}\left(p_{3}\right) K^{-}\left(p_{4}\right)$ with the $K^{*}$ exchange corresponding to Fig. 4 as

$$
\begin{aligned}
D_{t}= & \frac{-G^{2} / 2}{\left(p_{1}-p_{3}\right)^{2}-m_{K^{*}}^{2}}\left(p_{3} \cdot p_{4} p_{1} \cdot \epsilon_{2} p_{2} \cdot \epsilon_{1}\right. \\
& +p_{1} \cdot p_{2} p_{4} \cdot \epsilon_{1} p_{3} \cdot \epsilon_{2}+p_{1} \cdot p_{4} p_{2} \cdot p_{3} \epsilon_{1} \cdot \epsilon_{2} \\
& -p_{2} \cdot p_{3} p_{1} \cdot \epsilon_{2} p_{4} \cdot \epsilon_{1}-p_{1} \cdot p_{4} p_{2} \cdot \epsilon_{1} p_{3} \cdot \epsilon_{2} \\
& \left.-p_{1} \cdot p_{2} p_{3} \cdot p_{4} \epsilon_{1} \cdot \epsilon_{2}\right),
\end{aligned}
$$

and the $u$ channel

$$
D_{u}\left(p_{1}, p_{2}, p_{3}, p_{4}\right)=D_{t}\left(p_{1}, p_{2}, p_{4}, p_{3}\right) .
$$

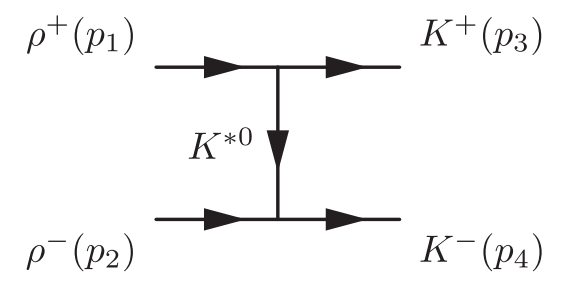

FIG. 4. $K^{*}$-exchange diagram for $\rho^{+} \rho^{-} \rightarrow K^{+} K^{-}$. 
Next we list the tree-level amplitudes for $\rho \rho \rightarrow K \bar{K}$ with the $K^{*}$-exchange as the following:

$$
\begin{aligned}
\rho^{+}\left(p_{1}\right) \rho^{-}\left(p_{2}\right) & \rightarrow K^{+}\left(p_{3}\right) K^{-}\left(p_{4}\right): D_{t}, \\
\rho^{+}\left(p_{1}\right) \rho^{-}\left(p_{2}\right) & \rightarrow K^{0}\left(p_{3}\right) \bar{K}^{0}\left(p_{4}\right): D_{u}, \\
\rho^{0}\left(p_{1}\right) \rho^{0}\left(p_{2}\right) & \rightarrow K^{+}\left(p_{3}\right) K^{-}\left(p_{4}\right): \frac{D_{t}+D_{u}}{2}, \\
\rho^{0}\left(p_{1}\right) \rho^{0}\left(p_{2}\right) & \rightarrow K^{0}\left(p_{3}\right) \bar{K}^{0}\left(p_{4}\right): \frac{D_{t}+D_{u}}{2} .
\end{aligned}
$$

Using Eqs. (7) and (18) we obtain the $I=0$ amplitude

$$
\begin{aligned}
T_{K^{*}}^{(0)}= & \frac{-\sqrt{6} G^{2} / 4}{\left(p_{1}-p_{3}\right)^{2}-m_{K^{*}}^{2}}\left(p_{3} \cdot p_{4} p_{1} \cdot \epsilon_{2} p_{2} \cdot \epsilon_{1}\right. \\
& +p_{1} \cdot p_{2} p_{4} \cdot \epsilon_{1} p_{3} \cdot \epsilon_{2}+p_{1} \cdot p_{4} p_{2} \cdot p_{3} \epsilon_{1} \cdot \epsilon_{2} \\
& -p_{2} \cdot p_{3} p_{1} \cdot \epsilon_{2} p_{4} \cdot \epsilon_{1}-p_{1} \cdot p_{4} p_{2} \cdot \epsilon_{1} p_{3} \cdot \epsilon_{2} \\
& \left.-p_{1} \cdot p_{2} p_{3} \cdot p_{4} \epsilon_{1} \cdot \epsilon_{2}\right)+\left(p_{3} \leftrightarrow p_{4}\right),
\end{aligned}
$$

which can be obtained from Eq. (14) by substituting $1 \rightarrow$ $\sqrt{6} / 4$ and $m_{\omega} \rightarrow m_{K^{*}}$.

\section{E. Partial-wave decomposition}

In term of these amplitudes with isospin $I=0$, we can calculate the partial-wave amplitudes in the $\ell S J I$ basis [5], denoted as $T_{\ell S ; \bar{\ell} \bar{S}}^{(J I)}(s)$ for the transition $(\bar{\ell} \bar{S} J I) \rightarrow$ $(\ell S J I)$

$$
\begin{aligned}
T_{\ell S ; \bar{\ell} \bar{S}}^{(J I)}(s)= & \frac{Y_{\bar{\ell}}^{0}(\hat{\mathbf{z}})}{\sqrt{2^{N}(2 J+1)}} \\
& \times \sum_{\substack{\sigma_{1}, \sigma_{2}, \bar{\sigma}_{1} \\
\bar{\sigma}, m}} \int \mathrm{d} \hat{\mathbf{p}}^{\prime \prime} Y_{\ell}^{m}\left(\hat{\mathbf{p}}^{\prime \prime}\right)^{*}\left(\sigma_{1} \sigma_{2} M \mid s_{1} s_{2} S\right) \\
& \times(m M \bar{M} \mid \ell S J)\left(\bar{\sigma}_{1} \bar{\sigma}_{2} \bar{M} \mid \bar{s}_{1} \bar{s}_{2} \bar{S}\right) \\
& \times(0 \bar{M} \bar{M} \mid \bar{\ell} \bar{S} J) T^{(I)}\left(p_{1}, p_{2}, p_{3}, p_{4}\right)
\end{aligned}
$$

with $M=\sigma_{1}+\sigma_{2}$ and $\bar{M}=\bar{\sigma}_{1}+\bar{\sigma}_{2}$. And $N$ accounts for identical particles, for example,

$$
\begin{array}{ll}
N=2 & \text { for } \rho \rho \rightarrow \pi \pi, \\
N=1 & \text { for } \rho \rho \rightarrow K \bar{K}
\end{array}
$$

By using Eq. (24) we can calculate the partial-wave projected tree-level amplitudes of Eqs. (8), (14), (19), and (23) with quantum numbers $I, \ell, S=0,0,0$. We denote $T_{00 ; 00}^{(00)}$ by $V$ for simplicity and we have

for $\rho \rho \rightarrow \pi \pi$ with the $\pi$ exchange

$V_{\pi}=\frac{2 g^{2}}{\sqrt{3}}\left(\frac{2\left(m_{\rho}^{2}-4 m_{\pi}^{2}\right)}{\sqrt{s-4 m_{\pi}^{2}} \sqrt{s-4 m_{\rho}^{2}}} \ln \frac{s-2 m_{\rho}^{2}+\sqrt{s-4 m_{\pi}^{2}} \sqrt{s-4 m_{\rho}^{2}}}{s-2 m_{\rho}^{2}-\sqrt{s-4 m_{\pi}^{2}} \sqrt{s-4 m_{\rho}^{2}}}+\frac{s}{m_{\rho}^{2}}+2\right)$,

for $\rho \rho \rightarrow \pi \pi$ with the $\omega$ exchange

$V_{\omega}=\frac{G^{\prime 2} s}{2 \sqrt{3}}\left(\frac{m_{\omega}^{2}}{\sqrt{s-4 m_{\pi}^{2}} \sqrt{s-4 m_{\rho}^{2}}} \ln \frac{s+2 m_{\omega}^{2}-2 m_{\pi}^{2}-2 m_{\rho}^{2}+\sqrt{s-4 m_{\pi}^{2}} \sqrt{s-4 m_{\rho}^{2}}}{s+2 m_{\omega}^{2}-2 m_{\pi}^{2}-2 m_{\rho}^{2}-\sqrt{s-4 m_{\pi}^{2}} \sqrt{s-4 m_{\rho}^{2}}}\right)$,

for $\rho \rho \rightarrow K \bar{K}$ with the $K$ exchange

$V_{K}=\frac{g^{2}}{2}\left(\frac{2\left(m_{\rho}^{2}-4 m_{K}^{2}\right)}{\sqrt{s-4 m_{K}^{2}} \sqrt{s-4 m_{\rho}^{2}}} \ln \frac{s-2 m_{\rho}^{2}+\sqrt{s-4 m_{K}^{2}} \sqrt{s-4 m_{\rho}^{2}}}{s-2 m_{\rho}^{2}-\sqrt{s-4 m_{K}^{2}} \sqrt{s-4 m_{\rho}^{2}}}+\frac{s}{m_{\rho}^{2}}+2\right)$,

for $\rho \rho \rightarrow K \bar{K}$ with the $K^{*}$ exchange

$V_{K^{*}}=\frac{G^{\prime 2} s}{4}\left(\frac{m_{K^{*}}^{2}}{\sqrt{s-4 m_{K}^{2}} \sqrt{s-4 m_{\rho}^{2}}} \ln \frac{s+2 m_{K^{*}}^{2}-2 m_{K}^{2}-2 m_{\rho}^{2}+\sqrt{s-4 m_{K}^{2}} \sqrt{s-4 m_{\rho}^{2}}}{s+2 m_{K^{*}}^{2}-2 m_{K}^{2}-2 m_{\rho}^{2}-\sqrt{s-4 m_{K}^{2}} \sqrt{s-4 m_{\rho}^{2}}}\right)$. 


\section{RESULTS AND DISCUSSION}

We label the three channels, $\rho \rho, K \bar{K}$, and $\pi \pi$ as 1,2 , and 3 , respectively. With the channel transition amplitudes $V_{\pi}$, $V_{\omega}, V_{K}$, and $V_{K^{*}}$ given in last section, we calculate the full amplitude and its pole positions by using the BetheSalpeter equation in its on-shell factorized form $[4,5]$

$$
T=\frac{V}{1-V G} .
$$

$G$ is a diagonal matrix made up by the two-point loop function $[4,5]$

$$
G_{j j}(s)=i \int \frac{d^{4} q}{(2 \pi)^{4}} \frac{1}{\left(q^{2}-m_{j}^{2}\right)\left((P-q)^{2}-m_{j}^{2}\right)}
$$

with $P$ the total four-momentum of the meson-meson systems and $q$ the loop momentum. The channel is labeled by the subindex $j$. By using dimensional regularization, the integration can be recast as

$$
G_{j j}(s)=\frac{1}{(4 \pi)^{2}}\left(a(\mu)+\log \frac{m_{j}^{2}}{\mu^{2}}+\sigma \log \frac{\sigma+1}{\sigma-1}\right)
$$

with

$$
\sigma=\sqrt{1-\frac{4 m_{j}^{2}}{s}}
$$

or using a momentum cutoff $q_{\max }$ as

$$
G_{j j}(s)=\frac{1}{2 \pi^{2}} \int_{0}^{q_{\max }} \mathrm{d} q \frac{q^{2}}{w\left(s-4 w^{2}+i \epsilon\right)}
$$

where $w=\sqrt{q^{2}+m_{j}^{2}}$. The integral can be done algebraically

$$
\begin{aligned}
G_{j j}(s)= & \frac{1}{(4 \pi)^{2}}\left\{\sigma \log \frac{\sigma \sqrt{1+\frac{m_{j}^{2}}{q_{\max }^{2}}}+1}{\sigma \sqrt{1+\frac{m_{j}^{2}}{q_{\max }^{2}}}-1}\right. \\
& \left.-2 \log \left[\frac{q_{\max }}{m_{j}}\left(1+\sqrt{1+\frac{m_{j}^{2}}{q_{\max }^{2}}}\right)\right]\right\} .
\end{aligned}
$$

Typical values of the cutoff $q_{\max }$ are around $1 \mathrm{GeV}$. $G_{j j}(s)$ has a right-hand cut above the threshold $2 m_{j}$. In order to make an analytical extrapolation to the second Riemann sheet we make use of the continuity property

$$
G_{j j}^{(2)}(\sqrt{s}+i \epsilon)=G_{j j}(\sqrt{s}-i \epsilon)
$$

where the index (2) indicates the second Riemann sheet of $G_{j j}$. Then

$$
\begin{aligned}
G_{j j}^{(2)}(\sqrt{s}+i \epsilon) & =G_{j j}(\sqrt{s}-i \epsilon) \\
& =G_{j j}(\sqrt{s}+i \epsilon)-2 i \operatorname{Im} G_{j j}(\sqrt{s}+i \epsilon) \\
& =G_{j j}(\sqrt{s}+i \epsilon)+\frac{i}{4 \pi} \frac{|\mathbf{p}|}{\sqrt{s}} .
\end{aligned}
$$

Other potentials of coupled channels like $\pi \pi-K \bar{K}$ can be found in [1]. Our results are shown in Table 1 for various $q_{\max }$ values. For comparison, the results for the $\rho \rho$ single channel without considering the coupled-channel effects as in Ref. [5] are shown in the second row. The $3 \sim 6$ rows show the results including one coupled channel with the exchanged meson listed in the first column. For example the $\pi$ denotes the $\rho \rho-\pi \pi$ channel with the $\pi$ exchange and so on. The seventh row gives the results including all three coupled channels of $\rho \rho, \pi \pi$, and $\bar{K} K$.

The above results show that the influence of the vector meson $\omega$ and $K^{*}$ exchanges is very small; the largest influence comes from the $\rho \rho-\pi \pi$ channel coupling by the pion exchange, which shifts up the pole mass and results in a sizable $\pi \pi$ decay width, comparable with relevant PDG values for $f_{0}(1500)$ [15]. For the $\rho \rho-K \bar{K}$ coupledchannel case we can see that the width is consistent with $f_{0}(1500)$ decaying into $K \bar{K}$ in PDG, which is about

TABLE I. Pole position for coupled channels.

\begin{tabular}{lcccc}
\hline \hline$q_{\max }(\mathrm{GeV})$ & 0.875 & 1.0 & 1.2 & 1.4 \\
\hline$\rho \rho$ only & 1494.8 & 1467.2 & 1427.3 & 1395.0 \\
$\pi$ & $1530.0-4.9 i$ & $1519.5-8.4 i$ & $1501.5-12.3 i$ & $1488.6-14.6 i$ \\
$\omega$ & $1492.2-0.7 i$ & $1466.5-1.0 i$ & $1428.1-1.1 i$ & $1400.0-1.1 i$ \\
$K$ & $1497.8-3.3 i$ & $1473.9-4.1 i$ & $1437.2-4.4 i$ & $1410.0-4.2 i$ \\
$K^{*}$ & $1489.6-0.5 i$ & $1463.3-0.5 i$ & $1424.5-0.4 i$ & $1396.1-0.3 i$ \\
3 channels & $1529.8-4.9 i$ & $1519.0-8.6 i$ & $1500.9-13.5 i$ & $1488.4-16.7 i$ \\
\hline \hline
\end{tabular}


8.9 MeV. When taking into account all the three channels, the pole position is close to the results by considering only the pion exchange contribution. With $q_{\max }=1.4 \mathrm{GeV}$, the pole mass and partial decay widths to $\pi \pi$ and $\bar{K} K$ are roughly consistent with PDG values for $f_{0}(1500)$. The largest decay channel should be $4 \pi$ either through $\rho \rho$ directly or by its cross talk with $\sigma \sigma$. Note that due to the binding energy of the molecule as well as the kinetic energy of $\rho$ inside the molecule, the $4 \pi$ decay width through the decay of $\rho$ inside the $\rho \rho$ molecule can be smaller than the decay width of a single free $\rho$ meson. A similar effect was pointed out by Refs. [16,17] in their studies of $d^{*}(2380)$ as a $\Delta \Delta$ molecule which gets a decay width smaller than the decay width of a single free $\Delta$ state. This kind of effect was also observed by the study of other hadronic molecules $[18,19]$.

In summary, the $\rho \rho$ scattering is revisited by including its coupled channels of pseudoscalar mesons, i.e., $\pi \pi$ and $\bar{K} K$. It is found that the coupled-channel effect is important and shifts up the pole mass of the dynamically generated scalar state significantly. It makes the state to be more consistent with $f_{0}(1500)$ rather than $f_{0}(1370)$ as favored by the previous studies $[4,5]$ without including these coupled channels. This leads to a nicely consistent picture with a recent dispersive study [20] where a new parametrization for the scalar pion form factors is derived by fitting it to
LHCb data on $\bar{B}_{s}^{0} \rightarrow J / \psi \pi \pi$ and finding an $f_{0}(1500)$ at mass $1465 \pm 18 \mathrm{MeV}$ coupling strongly to $\rho \rho$ (or $\sigma \sigma$ ). The $\rho \rho$ scattering has been extended to the $S$-wave interactions for the whole vector-meson nonet by two groups $[21,22]$. Both propose $f_{0}(1710)$ to be the $K^{*} \bar{K}^{*}$ dynamically generated state. We expect similar significant coupledchannel effects there. By including its coupled channels of pseudoscalar mesons, the $K^{*} \bar{K}^{*}$ dynamically generated state could be $f_{0}(1790)$ suggested by the BES data [23-25] instead of $f_{0}(1710)$. The $f_{0}(1370), f_{0}(1500)$, and $f_{0}(1710)$ have been studied before in the quarkoniaglueball mixing picture in Refs. [26-28], trying to pin down partial contributions of glueball, nonstrange, and strange quarkonia in these scalar mesons. With the new configuration of meson-meson dynamically generated states, the structure of these scalars should be richer than previous assumptions and deserve further exploration by expanding the configuration space.

\section{ACKNOWLEDGMENTS}

We thank Li-Sheng Geng, Feng-Kun Guo, Ulf-G. Meissner, and Eulogio Oset for helpful discussions. This project is supported by NSFC under Grant No. 11621131001 (CRC110 cofunded by DFG and NSFC) and Grant No. 11835015.
[1] J. A. Oller and E. Oset, Nucl. Phys. A620, 438 (1997); A652, 407(E) (1999).

[2] E. Oset and A. Ramos, Nucl. Phys. A635, 99 (1998).

[3] J. A. Oller and U. G. Meissner, Phys. Lett. B 500, 263 (2001).

[4] R. Molina, D. Nicmorus, and E. Oset, Phys. Rev. D 78, 114018 (2008).

[5] D. Gülmez, U.-G. Meißner, and J. A. Oller, Eur. Phys. J. C 77, 460 (2017).

[6] J. A. Oller and E. Oset, Phys. Rev. D 60, 074023 (1999).

[7] M. Albaladejo and J. A. Oller, Phys. Rev. C 84, 054009 (2011).

[8] M. Albaladejo and J. A. Oller, Phys. Rev. C 86, 034005 (2012).

[9] Z. H. Guo, J. A. Oller, and G. Ríos, Phys. Rev. C 89, 014002 (2014).

[10] J. A. Oller, Phys. Rev. C 93, 024002 (2016).

[11] L. S. Geng, R. Molina, and E. Oset, Chin. Phys. C 41, 124101 (2017).

[12] M. Bando, T. Kugo, S. Uehara, K. Yamawaki, and T. Yanagida, Phys. Rev. Lett. 54, 1215 (1985).

[13] M. Bando, T. Kugo, and K. Yamawaki, Phys. Rep. 164, 217 (1988).

[14] H. Nagahiro, L. Roca, A. Hosaka, and E. Oset, Phys. Rev. D 79, 014015 (2009).
[15] M. Tanabashi et al. (Particle Data Group), Phys. Rev. D 98, 030001 (2018).

[16] J. A. Niskanen, Phys. Rev. C 95, 054002 (2017).

[17] A. Gal, Phys. Lett. B 769, 436 (2017).

[18] Y.H. Lin and B.S. Zou, Phys. Rev. D 98, 056013 (2018).

[19] Y. H. Lin, C. W. Shen, and B. S. Zou, Nucl. Phys. A980, 21 (2018).

[20] S. Ropertz, C. Hanhart, and B. Kubis, Eur. Phys. J. C 78, 1000 (2018).

[21] L. S. Geng and E. Oset, Phys. Rev. D 79, 074009 (2009).

[22] M. L. Du, D. Gülmez, F. K. Guo, U. G. Meissner, and Q. Wang, Eur. Phys. J. C 78, 988 (2018).

[23] M. Ablikim et al. (BES Collaboration), Phys. Lett. B 607, 243 (2005).

[24] M. Ablikim et al. (BES Collaboration), Phys. Lett. B 598, 149 (2004).

[25] M. Ablikim et al. (BES Collaboration), Phys. Lett. B 603, 138 (2004).

[26] F. E. Close and Q. Zhao, Phys. Rev. D 71, 094022 (2005).

[27] F. Giacosa, T. Gutsche, V. E. Lyubovitskij, and A. Faessler, Phys. Rev. D 72, 094006 (2005); Phys. Lett. B 622, 277 (2005).

[28] P. Chatzis, A. Faessler, T. Gutsche, and V. E. Lyubovitskij, Phys. Rev. D 84, 034027 (2011). 